\title{
Panel of Microsatellite Markers
}

\section{for Whole-Genome Scans and Radiation Hybrid Mapping and a Mouse Family Tree}

\author{
Leonard C. Schalkwyk, ${ }^{1}$ Martin Jung, ${ }^{2,3}$ Angelika Daser, ${ }^{4}$ Michael Weiher, ${ }^{5}$ \\ Jörn Walter, Heinz Himmelbauer, and Hans Lehrach \\ ${ }^{1}$ Max-Planck-Institut für Molekulare Genetik, Berlin, Germany; ${ }^{2}$ Mikrosatellitenzentrum, Max-Delbrück-Centrum, \\ D-14059 Berlin, Germany; ${ }^{4}$ Institute of Laboratory Medicine and Pathobiochemistry Charité, Campus Virchow, \\ D-13353 Berlin, Germany
}

\begin{abstract}
To facilitate whole-genome scan experiments, we selected a panel of 128 microsatellite markers on the basis of spacing and polymorphism in the strains DBA/2, BALB/C, AKR, C57BL/6, C57BL/10, A/], C3H, 129/], SJL/], JF1, and PWB. Many of the primer pairs were redesigned for better performance. The last four strains were not characterized previously using these markers. JF1 and PWB are particularly interesting for intersubspecific crosses offering high polymorphism. We provide allele size data for the markers on these strains and add them to the emerging radiation hybrid framework map, which is not continuous except for chromosome 17 and 13. Information on the interrelationships of strains is useful both because of the importance of polymorphism in designing crosses and the background in assessing phenotypes. Microsatellites offer a widely dispersed, selectively neutral set of characters that lends itself conceptually to parsimony methods of analysis. The microsatellite allele size data were recoded as binary discrete characters in such a way that adjacent sizes differ by one step. Trees were generated using a Wagner parsimony method. As expected, the non-Mus domesticus strains, PWB (musculus) and JF1 (molossinus), are excluded from the domesticus strains. Among the domesticus strains, $\mathrm{C} 57 \mathrm{BL} / 6$ and $\mathrm{C} 57 \mathrm{BL} / 10$ (derived from the same founding pair) form a strongly supported group, as do $\mathrm{C} 3 \mathrm{H}$, $A /$ ], and $B A L B / C$ (derived from the Bagg albino stock). No unique branching order for SJL/], AKR, and DBA/2 is strongly supported, which may reflect a complicated history. Strain 129/] is clearly placed as the most deeply diverged of the domesticus strains represented.
\end{abstract}

To map quantitative trait loci it is necessary to test the genotypes of cross progeny with a panel of polymorphic markers spaced at 10 - to 20 -cM intervals. For example, with 52 backcross animals, linkage across $15 \mathrm{cM}$ can be detected at 95\% confidence. Therefore, in principle, a set of markers no more than $15 \mathrm{cM}$ from chromosome ends and $30 \mathrm{cM}$ from each other would suffice (Silver 1995). Although there are now many microsatellite markers available (primarily MIT markers from Dietrich et al. 1996) and the average density is about four markers/cM, it is still often difficult to find sufficient markers with differing alleles for a particular pair of inbred strains. This is especially true if these are both derived from Mus musculus domesticus. In addition, although the quality of published microsatellite markers is good, most of them are mass-produced and have not been extensively characterized, and some primers are not robust. For the Dietrich et al. (1996) markers, allele size data are available for 12 strains, including a Mus

\footnotetext{
${ }^{1}$ Corresponding author.

E-MAIL schalkwy@mpimg-berlin-dahlem.mpg.de; FAX 4930 84131380.

Present addresses: ${ }^{3}$ Ingenium Pharmaceuticals AG, D-82152 Martinsried, Germany; ${ }^{5}$ Genome Pharmaceuticals Corporation AG (GPC AG), Martinsried, Germany.
}

spretus and a Mus musculus castaneus strain. However, for any given pair of strains it is a substantial job to identify a suitable marker panel and verify that these are detectably polymorphic.

We have selected a set of highly polymorphic markers for the Mus musculus strains DBA/2, BALB/c, AKR, C57BL/6, A/J, and C3H (typed by Dietrich et al. 1996), redesigned many of the primers, and tested them on these strains, as well as C57BL/10, SJL/J, 129/J, JF1, and PWB. PWB is an inbred strain derived from a wild-derived Czech Mus musculus musculus isolate (Forejt 1991). JF1 is a recently established inbred strain of Japanese Mus musculus molossinus origin (a natural hybrid of M. m. musculus and M. m. castaneus; Bonhomme and Guénet 1996), which should also differ at many loci from the standard strains (Koide et al. 1998). The remaining strains are classic inbred strains that are quite closely related (Silver 1995). 129/J in particular is a widely used strain for which allele size data are not provided in the MIT data set. Data for 209 simple sequence-length polymorphisms (SSLPs) on strain 129 were published recently (Matin et al. 1998), only 2 of which are in common with the work reported here.

It would be useful to have a clear picture of the 
degrees of relatedness of mouse strains to each other. The breeding histories of the classical inbred strains are to some extent known (Festing 1996), but there are little data on the outbred progenitor populations. The relationships of the inbred strains of mice were investigated using parsimony by Atchley and Fitch (1991), employing classical genotypes at 144 loci over the whole genome. At each locus, possession by two strains of the same-sized allele indicates probable similarity by descent of that sequence. Using many loci spread over the genome, it should be possible to reconstruct a phylogeny of the genome as a whole, at least to the extent that the history of these strains can be represented by a tree (i.e., no recent interbreeding, which would give a net). Atchley and Fitch (1991) obtained a tree that reflected the groups of strains known to be closely related: the C57 and C58 strains, and the Bagg albino descendants $\mathrm{BALB} / \mathrm{c}, \mathrm{A}$, and $\mathrm{C} 3 \mathrm{H}$. A somewhat deeper clade united many of the others, including SJL, DBA, and AKR. The main findings were reproduced by a subset of the data identified as "protein loci" but not by smaller subsets of "immune" and "viral." One discrepancy between the overall and protein trees is the position of strain 129, which is a specific relative of the C57 and C58 group in the former and of the SJL/DBA/AKR group in the latter.

Radiation hybrid $(\mathrm{RH})$ panels are an attractive tool for mapping arbitrary sequences (such as ESTs) without the need for polymorphism and high resolution, and have been important in making a very gene-rich human map (Deloukas et al. 1998). The T31 panel has been characterized sufficiently to indicate that it will be a useful mapping tool. Typings of 472 markers on the panel are available from the public database (http://www.jax.org/resources/documents/cmdata/ rhmap/rhdata.html). This database includes the initial characterization (McCarthy et al. 1997) and detailed maps of chromosome 17 (Schalkwyk et al. 1998) and chromosome 13 (Elliott et al. 1999). However, these data do not provide a framework suitably dense to allow unknown markers to be mapped with certainty. We have typed the T31 RH panel (McCarthy et al. 1997) with the same panel of markers and thus contributed to an emerging map framework for this panel.

\section{RESULTS AND DISCUSSION}

The panel of microsatellite markers was selected using published genetic positions and allele sizes (Breen et al. 1994; Dietrich et al. 1996) with two objectives: maximizing polymorphism in our chosen list of strains, and approximating a 10-cM spacing. This resulted in a preliminary list of 148 markers, 114 of which were selected. The remaining markers did not produce the expected PCR performance, polymorphism, or position. Another 14 markers were selected to patch the resulting gaps. Primer sequences were inspected for potential problems and redesigned where possible to eliminate cases where the $3^{\prime}$ end contained one or more CA dinucleotide sequences, which experience indicates leads to stuttering artifacts. Other primer sequences were redesigned to adjust the sizes of the products to facilitate multiplexing of the markers within chromosomes.

\section{Mouse Strain Panel Data}

The 11 strains tested here (allele size data presented in Table 1) give rise to 55 pairs of strains that could be considered for a cross. For different pairs, the present work provides between 31 and 120 polymorphic markers (Table 2) - in many cases, a nearly complete, tested panel of markers. The allele size data, as well as lists of polymorphic markers for each possible pair, are available at http://www.mpimg-berlin-dahlem.mpg.de/ $\sim$ rodent/bin/polymarkerleo.cgi. These markers were also tested on M. spretus strain SMZ. The animal tested was heterozygous at 32 of the loci (data not shown).

Allele sizes for seven of the strains were also estimated by Dietrich et al. (1996), using conventional sequencing acrylamide gels on which ${ }^{32} \mathrm{P}$ kinase-labeled PCR products were separated. This method, using size standards run separately, is likely to be less accurate for absolute sizes than the fluorescent labeling and internal standard method used here. Nonetheless, relative fragment sizes should be robust. Taking this into account, there is agreement in allele sizes. There is perfect agreement for only 4 markers, and in 28 other cases the relative sizes of the alleles agree, but the absolute sizes differ. In 16 cases, one allele size disagrees, and in 13 cases two allele sizes disagree. These categories are indicated in the last column of Table 1 and are adjusted in those cases where redesigned primers change the expected product size. In the cases of C57BL/6, C57BL/ $10, \mathrm{AKR}$, and $\mathrm{A} / \mathrm{J}$, some fraction of the differences may be attributed to mutations occurring since the divergence of the different sublines used in the two studies.

The number of polymorphic markers follows the known relatedness of the strains in question (Table 2). The highest numbers come from the pairs involving PWB and JF1, reflecting the divisions between the three subspecies musculus (PWB), molossinus (JF1), and domesticus (the remaining strains). At the opposite extreme are the highly similar strains C57BL/6 and C57BL/10 (both derived from the same founding pair) and the group BALB/c, C3H, A/J, which are all derived from the Bagg Albino stock (Festing 1996).

\section{Microsatellite Tree}

Because inbred mouse strains are derived from a common heritage in the M. musculus complex species and have not been allowed to interbreed, it is plausible to treat them as if they were species and it is interesting to determine whether their history can be reconstructed. 


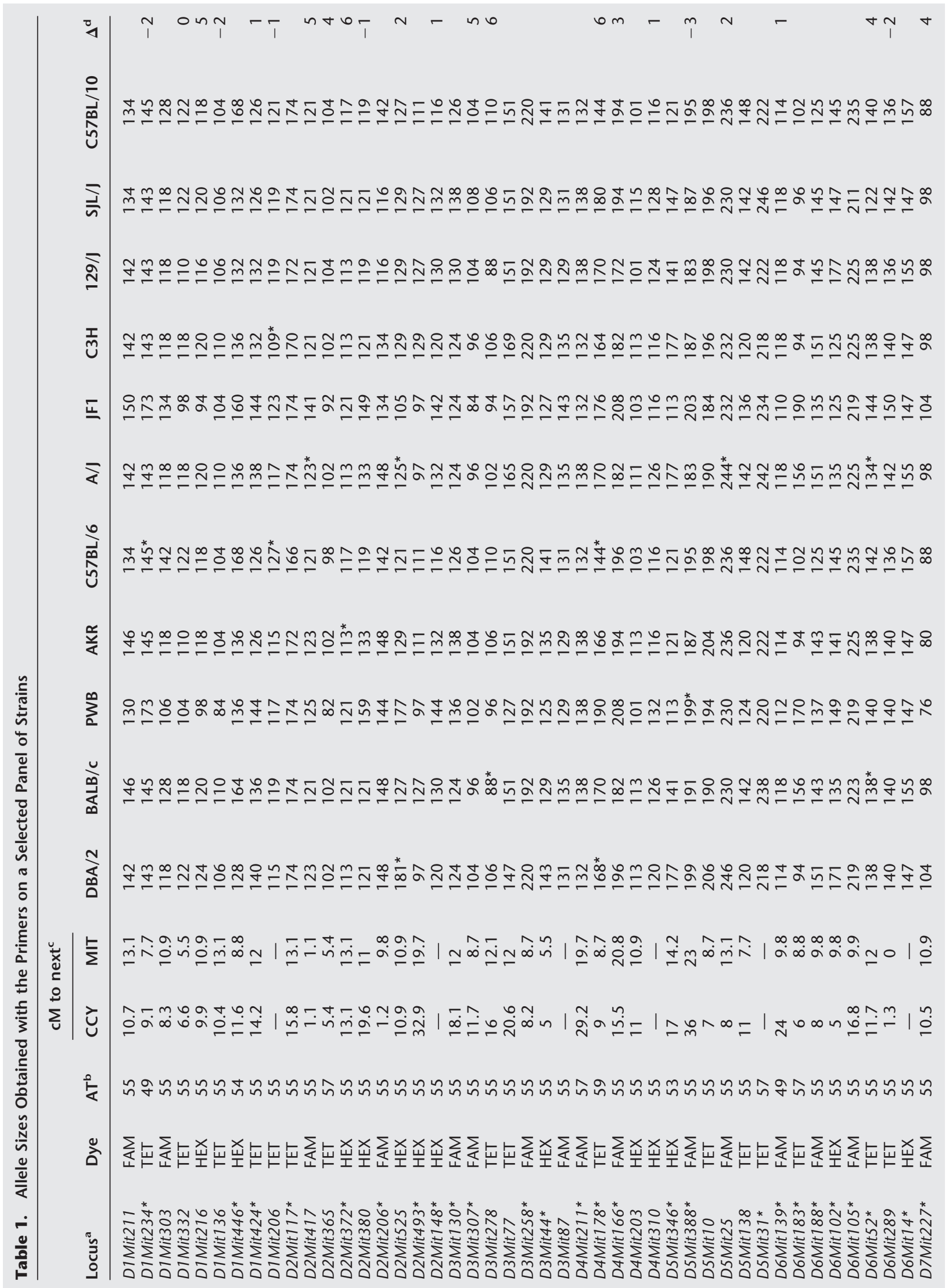

880 Genome Research

www.genome.org 
Downloaded from genome.cshlp.org on April 25, 2023 - Published by Cold Spring Harbor Laboratory Press

Mouse Microsatellite Panel and Tree

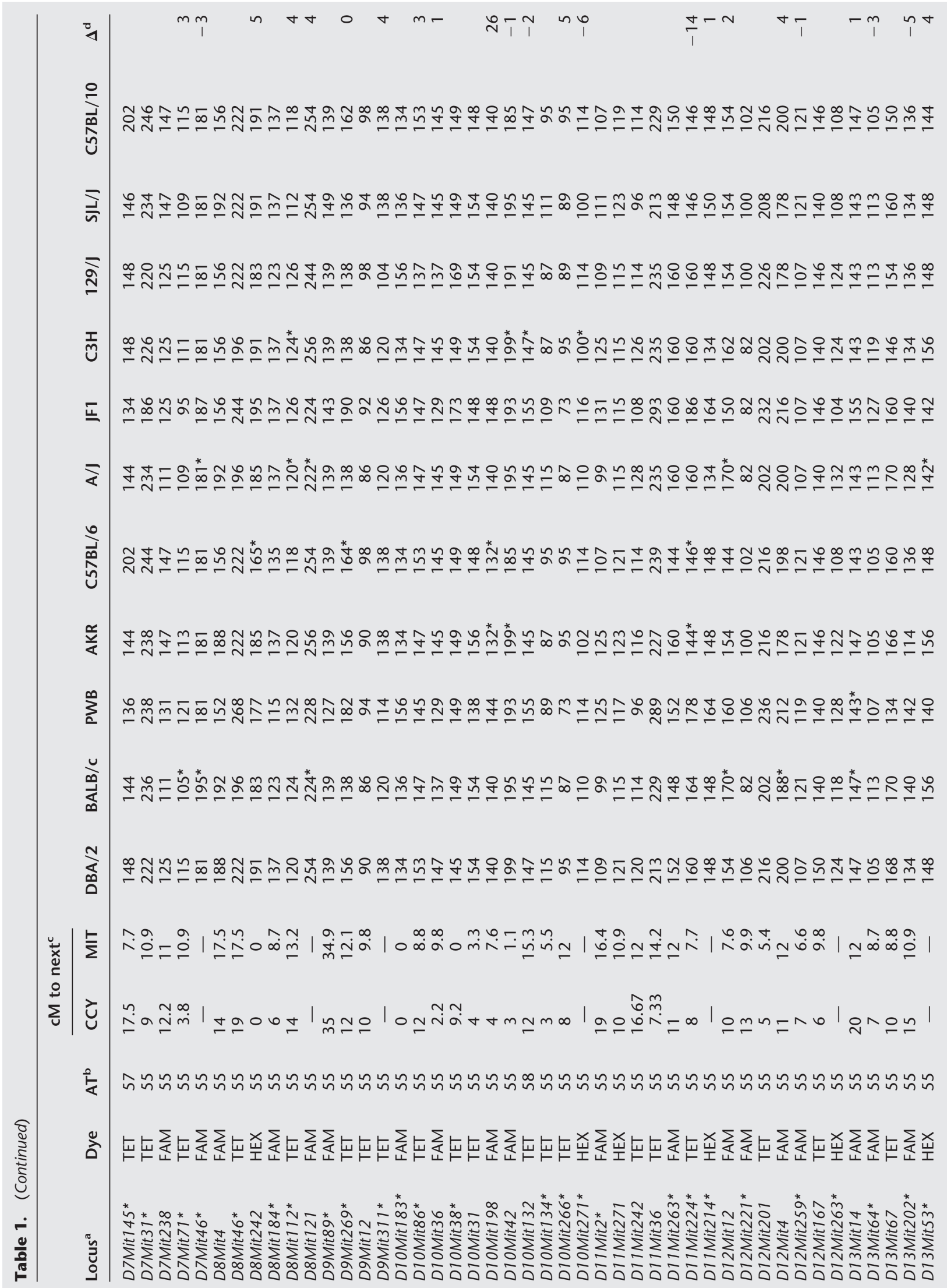




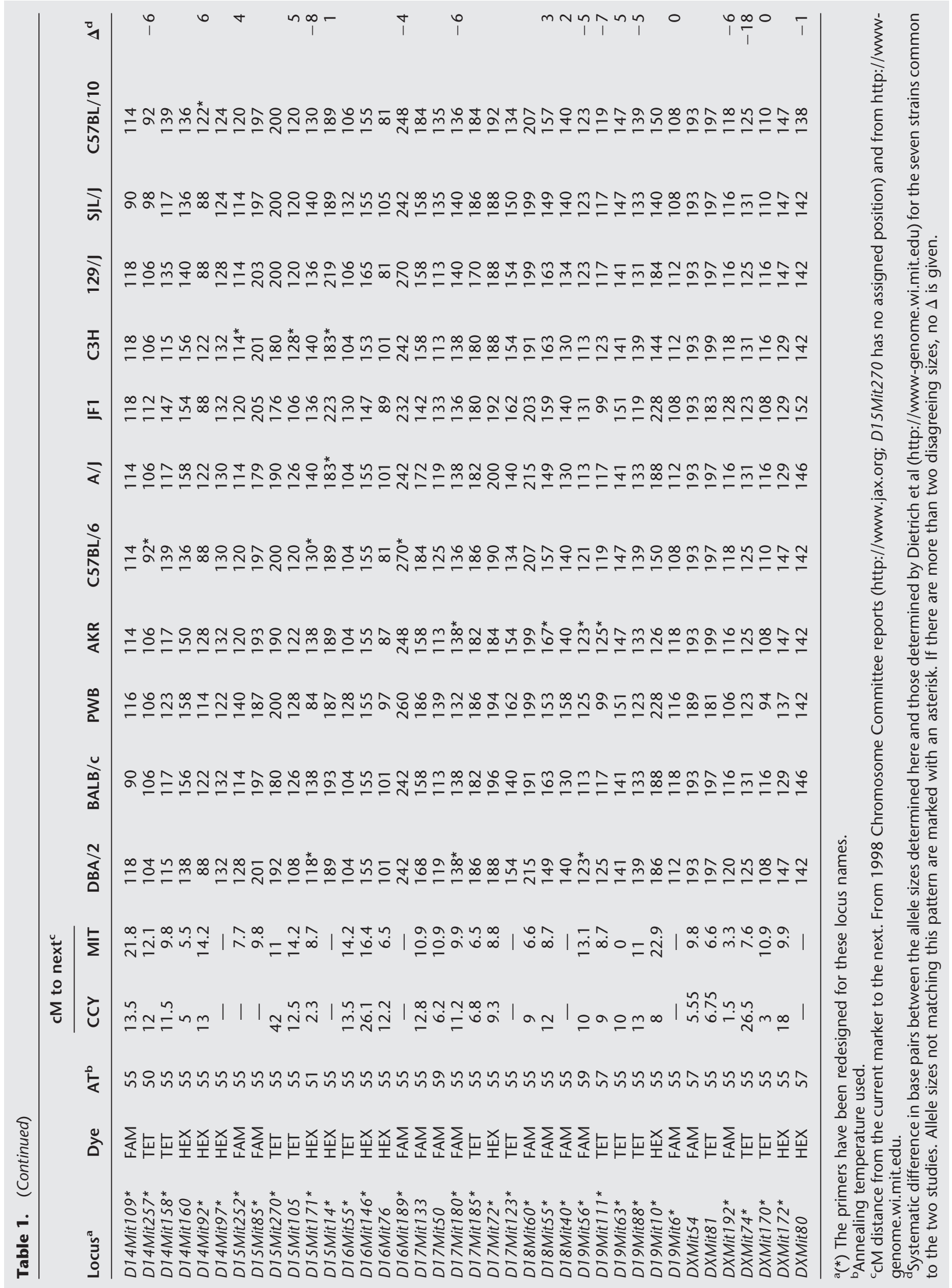

882 Genome Research

www.genome.org 
Table 2. Number of Polymorphic Markers in the Panel for Each Possible Pair of Strains

\begin{tabular}{|c|c|c|c|c|c|c|c|c|c|c|}
\hline & C57BL/10 & AKR & $\mathrm{DBA} / 2$ & $\mathrm{C} 3 \mathrm{H}$ & $A / J$ & SJL/J & $129 / J$ & $\mathrm{BALB} / \mathrm{c}$ & JF1 & PWB \\
\hline $\mathrm{C} 57 \mathrm{BL} / 6$ & 31 & 89 & 94 & 111 & 115 & 92 & 98 & 114 & 114 & 120 \\
\hline $\mathrm{C} 57 \mathrm{BL} / 10$ & & 87 & 92 & 108 & 114 & 90 & 98 & 109 & 114 & 120 \\
\hline AKR & & & 82 & 89 & 96 & 88 & 93 & 94 & 115 & 114 \\
\hline $\mathrm{DBA} / 2$ & & & & 76 & 93 & 93 & 93 & 105 & 112 & 115 \\
\hline $\mathrm{C} 3 \mathrm{H}$ & & & & & 68 & 94 & 87 & 76 & 108 & 117 \\
\hline$A / J$ & & & & & & 88 & 94 & 56 & 116 & 116 \\
\hline $\mathrm{SJL} / J$ & & & & & & & 84 & 87 & 117 & 111 \\
\hline $129 /$ & & & & & & & & 89 & 116 & 116 \\
\hline $\mathrm{BALB} / \mathrm{C}$ & & & & & & & & & 116 & 118 \\
\hline JF1 & & & & & & & & & & 107 \\
\hline
\end{tabular}

One important issue for which this information gives some insight is the effect of genetic background in assessing phenotypes. Microsatellites are a dispersed set of selectively neutral, highly variable samples from across the genome. Although collected for a different purpose they offer an outstanding source of information about strain histories. Given sufficient data, the histories of specific portions of the genome could probably be reconstructed, which would not necessarily be the same as the whole-genome average derived here.

Microsatellite data lend itself conceptually to parsimony analysis because alleles differ by discrete steps, and in those cases where it has been observed directly, they mutate predominantly by gain or loss of one repeat unit (Weber and Wong 1993; Amos et al. 1996; Primmer et al. 1998). Compared to methods based on a distance matrix, parsimony allows more information to be considered in the analysis (specific mutation steps rather than simple proportion of alleles of the same size).

To do the parsimony analysis with PHYLIP 3.5c (Felsenstein 1989) which accepts only binary discrete characters, the microsatellite allele size data were recoded as $n-1$ binary discrete characters, where $n$ is the number of alleles (ranging from 2 to 9), in such a way that adjacent sizes differ by one step. This gives an order to the mutational changes between alleles but does not presuppose that a particular allele is ancestral. Thus, for example, if a primer pair gives products sized 102,104 , and 108 bp on our panel of strains, the alleles would be coded using two bits: 00,01 , and 11 , respectively. This yields a vector (binary string) of length 520 for each strain. Wagner parsimony analysis was done with the MIX program of PHYLIP (Felsenstein 1989). We tested the robustness of the results by running the analysis on 100 different subsamples of the data (jackknife replicates generated with seqboot), and the consensus tree is shown in Figure 1 . The Wagner parsimony method does not assume that the ancestral state is known or that mutation in one direction is more probable than the other. It does, however, assume that species and characters both evolve independently, which perhaps cannot be said of multiple characters (bits) derived from a single microsatellite. Nonetheless, the tree derived is largely congruent with expectations from the known history of the strains and with the analysis of Atchley and Fitch (1991) of 144 single locus genotypes. Most of the branches are highly robust as indicated by jackknife resampling, showing that the observed branching order is not the result of a subset of the recoded data. As expected, the non-domesticus strains, PWB (musculus) and JF1 (molossinus), are distant from each other and from the remaining domesticus strains. Among the domesticus strains, C57BL/6 and $\mathrm{C} 57 \mathrm{BL} / 10$ (derived from the same founding pair) form a strongly supported group, as do $\mathrm{C} 3 \mathrm{H}, \mathrm{A} / \mathrm{J}$, and BALB/ c. No unique branching order for $S J L / J, A K R$, and $\mathrm{DBA} / 2$ is supported strongly, which may reflect a more complicated history. Strain $129 / \mathrm{J}$ is clearly placed as the most deeply diverged of the domesticus strains represented. In fact these results provide no evidence that

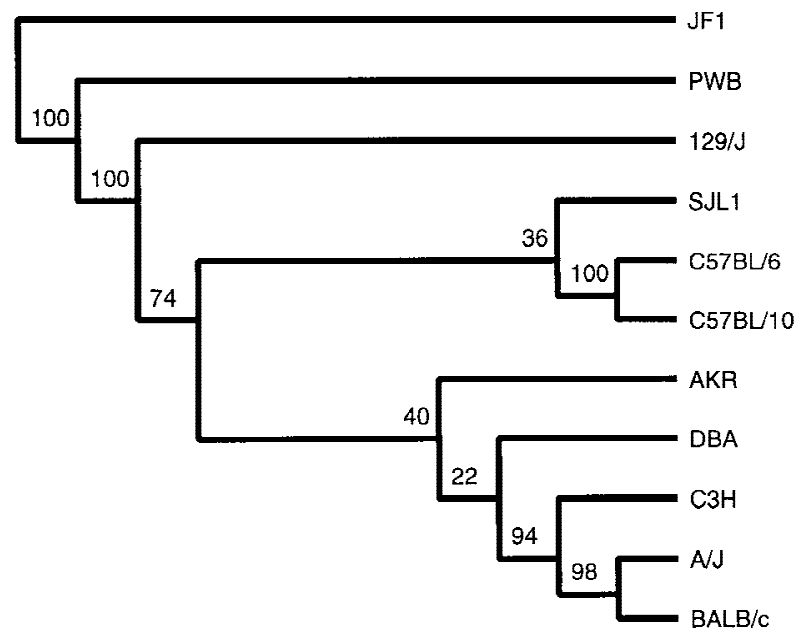

Figure 1 Unrooted consensus tree (of 100 jackknife replicates) derived from microsatellite allele size data by parsimony analysis. Numbers at the forks represent the number of times the group to the right of the fork occurred in 100 jackknife (sampling without replacement) samplings of the data. Branch lengths do not represent distances. 
Schalkwyk et al.

Table 3. Summary of RH Results

\begin{tabular}{|c|c|c|c|c|c|c|c|c|c|}
\hline Locus & Pos. & Prev. locus & Pos. & lod & cR & Next locus & Pos. & lod & CR \\
\hline D1Mit211 & 15 & D1Mit316 & 8 & 1.5 & 108.2 & D1Mit171 & 20.2 & 3.3 & 75.7 \\
\hline D1Mit234 & 26 & D1Mit232 & 21 & 6.3 & 51.4 & D1Mit236 & 26 & 11.7 & 25.1 \\
\hline D1Mit303 & 35 & D1Mit236 & 26 & 2.7 & 86.0 & D1Mit19 & 37 & 3.6 & 73.8 \\
\hline D1Mit332 & 43 & D1Mit46 & 43 & 5.8 & 53.8 & D1Mit216 & 50 & 5.8 & 56.0 \\
\hline D1Mit216 & 50 & D1Mit332 & 43 & 5.9 & 56.0 & D1Mit10 & 57 & 2.7 & 78.3 \\
\hline D1Mit1 36 & 60 & D1Mit10 & 57 & 3.9 & 64.6 & D1Mit87 & 62 & 5.2 & 57.1 \\
\hline D1Mit446 & 70 & D1Mit286 & 67 & 10.0 & 30.6 & D1Mit102 & 73 & 7.5 & 38.9 \\
\hline D1Mit424 & 82 & D1Mit102 & 73 & 5.6 & 50.3 & D1Mit33 & 82 & 10.6 & 28.1 \\
\hline D1Mit206 & 96 & D1Mit36 & 92 & 6.4 & 50.3 & D1Mit291 & 102 & 6.5 & 48.3 \\
\hline D2Mit1 17 & 5 & D2Mit312 & 2 & 3.5 & 71.7 & D2Mit149 & 7 & 5.0 & 60.1 \\
\hline D2Mit417 & 22 & D2Mit149 & 7 & 2.3 & 90.9 & D2Mit365 & 22 & 15.0 & 19.9 \\
\hline D2Mit365 & 22 & D2Mit417 & 21 & 15.0 & 19.9 & D2Mit372 & 27 & 1.7 & 103.8 \\
\hline D2Mit372 & 27 & D2Mit365 & 22 & 1.8 & 103.8 & D2Mit297 & 29 & 4.1 & 66.8 \\
\hline D2Mit380 & 40 & D2Mit182 & 37 & 4.0 & 68.9 & D2Mit44 & 53 & 3.1 & 78.1 \\
\hline D2Mit206 & 60 & D2Mit300 & 53 & 7.4 & 42.9 & D2Mit274 & 62 & 6.0 & 49.9 \\
\hline D2Mit525 & 61 & D2Mit106 & 76 & 17.2 & 10.0 & D2Mit194 & 81 & 7.6 & 39.6 \\
\hline D2Mit493 & 72 & D2Mit285 & 86 & 12.3 & 22.4 & D2Mit263 & 92 & 10.9 & 23.9 \\
\hline D2Mit148 & 105 & D2Mit263 & 92 & 7.8 & 36.8 & D2Mit346 & 92 & 13.7 & 19.6 \\
\hline D3Mit130 & 4 & D3Mit264 & 4 & 10.6 & 28.6 & D3Mit203 & 11 & 1.3 & 117.8 \\
\hline D3Mit307 & 22 & D3Mit224 & 22 & 3.8 & 65.4 & D3Mit22 & 34 & 0.0 & 230.3 \\
\hline D3Mit278 & 25 & D3Mit22 & 34 & 6.9 & 48.5 & D3Mit212 & 40 & 4.3 & 67.1 \\
\hline D3Mit77 & 37 & D3Mit29 & 45 & 1.8 & 103.0 & D3Mit215 & 55 & 1.5 & 105.6 \\
\hline D3Mit258 & 70 & D3Mit42 & 59 & 3.6 & 73.6 & D3Mit84 & 72 & 12.4 & 23.4 \\
\hline D3Mit44 & 79 & D3Mit200 & 77 & 19.4 & 8.0 & D3Mit59 & 84 & 7.2 & 48.5 \\
\hline D3Mit87 & 84 & D3Mit59 & 84 & 14.0 & 20.2 & D3Mit163 & 88 & 11.0 & 30.0 \\
\hline D4Mit211 & 6 & D4Mit149 & 12 & 4.6 & 2.3 & D4Mit94 & 11 & 2.8 & 74.4 \\
\hline D4Mit178 & 36 & D4Mit111 & 22 & 1.5 & 84.2 & D4Mit245 & 43 & 6.5 & 50.6 \\
\hline D4Mit166 & 45 & D4Mit245 & 42 & 5.9 & 55.3 & D4Mit9 & 45 & 13.6 & 22.6 \\
\hline D4Mit203 & 60 & D4Mit12 & 57 & 4.6 & 51.8 & D4Mit54 & 66 & 7.6 & 43.2 \\
\hline D4Mit310 & 71 & D4Mit54 & 66 & 8.7 & 38.9 & D4Mit225 & 80 & 2.4 & 79.2 \\
\hline D5Mit346 & 1 & - & - & - & - & D5Mit66 & 17 & 0.0 & 237.5 \\
\hline D5Mit388 & 18 & D5Mit66 & 17 & 5.4 & 58.5 & D5Mit78 & 26 & 4.0 & 69.7 \\
\hline D5Mit10 & 54 & D5Mit200 & 36 & 2.6 & 86.3 & D5Mit157 & 57 & 6.6 & 50.8 \\
\hline D5Mit25 & 61 & D5Mit240 & 59 & 7.2 & 45.4 & D5Mit136 & 65 & 8.4 & 37.4 \\
\hline D5Mit1 38 & 69 & D5Mit95 & 68 & 4.2 & 65.8 & D5Mit221 & 80 & 1.1 & 110.9 \\
\hline D5Mit31 & 80 & D5Mit221 & 80 & 14.5 & 15.2 & D5Mit292 & 83 & 13.2 & 22.8 \\
\hline D6Mit1 39 & 3 & D6Mit 138 & 1 & 11.8 & 26.3 & D6Mit223 & 19 & 1.6 & 102.7 \\
\hline D6Mit188 & 33 & D6Mit123 & 29 & 4.8 & 61.3 & D6Mit8 & 36 & 2.7 & 79.4 \\
\hline D6Mit102 & 41 & D6Mit31 & 39 & 16.2 & 14.2 & D6Mit105 & 46 & 4.2 & 67.5 \\
\hline D6Mit105 & 47 & D6Mit102 & 41 & 4.3 & 67.5 & D6Mit256 & 60 & 4.4 & 59.6 \\
\hline D6Mit52 & 61 & D6Mit24 & 57 & 7.0 & 46.0 & D6Mit289 & 62 & 17.7 & 14.6 \\
\hline D6Mit289 & 62 & D6Mit52 & 61 & 17.7 & 14.6 & Kap & 64 & 6.5 & 46.0 \\
\hline D6Mit 14 & 74 & D6Mit259 & 67 & 10.7 & 25.5 & D6Mit15 & 74 & 9.4 & 32.2 \\
\hline D7Mit227 & 16 & D7Mit246 & 15 & 5.1 & 58.2 & D7Mit69 & 25 & 3.7 & 66.9 \\
\hline D7Mit145 & 27 & D7Mit69 & 25 & 8.0 & 39.2 & D7Mit232 & 27 & 10.3 & 30.9 \\
\hline D7Mit31 & 44 & D7Mit 176 & 27 & 3.6 & 71.3 & D7Mit220 & 52 & 2.4 & 90.1 \\
\hline D7Mit238 & 53 & D7Mit220 & 52 & 5.0 & 60.0 & D7Mit 165 & 64 & 3.7 & 70.5 \\
\hline D7Mit71 & 65 & D7Mit 165 & 64 & 1.3 & 108.2 & D7Mit 166 & 66 & 2.4 & 89.4 \\
\hline D7Mit46 & 69 & D7Mit12 & 66 & 6.5 & 48.1 & - & - & - & - \\
\hline D8Mit4 & 14 & D8Mit223 & 11 & 4.9 & 61.8 & D8Mit191 & 21 & 9.9 & 30.5 \\
\hline D8Mit46 & 28 & D8Mit227 & 24 & 0.7 & 125.5 & D8Mit261 & 33 & 0.5 & 146.1 \\
\hline D8Mit242 & 47 & D8Mit184 & 47 & 23.0 & 5.2 & D8Mit112 & 53 & 0.4 & 163.0 \\
\hline D8Mit184 & 47 & D8Mit183 & 47 & 11.0 & 23.4 & D8Mit242 & 47 & 23.0 & 5.2 \\
\hline D8Mit1 12 & 53 & D8Mit242 & 47 & 0.5 & 163.0 & D8Mit12 & 53 & -0.1 & 274.9 \\
\hline D8Mit121 & 67 & D8Mit13 & 67 & 20.4 & 5.8 & D8Mit93 & 72 & 5.6 & 50.3 \\
\hline D9Mit89 & 8 & D9Mit64 & 7 & 14.3 & 11.7 & D9Mit90 & 9 & 15.9 & 14.4 \\
\hline D9Mit269 & 43 & D9Mit207 & 33 & 0.6 & 122.8 & D9Mit196 & 48 & 6.4 & 48.9 \\
\hline D9Mit12 & 55 & D9Mit136 & 54 & 21.3 & 5.5 & D9Mit 182 & 55 & 12.0 & 23.4 \\
\hline D9Mit311 & 65 & D9Mit24 & 56 & 5.7 & 57.3 & D9Mit52 & 72 & 8.8 & 35.0 \\
\hline D10Mit183 & 17 & D10Mit86 & 17 & 9.0 & 37.8 & D10Mit184 & 26 & 1.8 & 113.6 \\
\hline D10Mit86 & 17 & D10Mit16 & 16 & 5.3 & 57.8 & D10Mit183 & 17 & 9.0 & 37.8 \\
\hline D10Mit36 & 29 & D10Mit38 & 27 & 7.5 & 47.5 & D10Mit20 & 32 & 3.6 & 74.9 \\
\hline D10Mit38 & 27 & D10Mit184 & 26 & 10.6 & 29.8 & D10Mit36 & 29 & 7.4 & 47.5 \\
\hline D10Mit31 & 36 & D10Mit20 & 32 & 15.2 & 13.9 & D10Mit42 & 44 & 1.9 & 101.6 \\
\hline D10Mit42 & 44 & D10Mit31 & 36 & 2.0 & 101.6 & D10Mit132 & 47 & 10.0 & 34.7 \\
\hline D10Mit132 & 47 & D10Mit42 & 44 & 10.1 & 34.7 & D10Mit117 & 48 & 13.6 & 20.7 \\
\hline
\end{tabular}


Table 3. (Continued)

\begin{tabular}{|c|c|c|c|c|c|c|c|c|c|}
\hline Locus & Pos. & Prev. locus & Pos. & lod & $\mathbf{c R}$ & Next locus & Pos. & lod & cR \\
\hline D10Mit134 & 59 & D10Mit95 & 51 & 4.3 & 64.3 & D10Mit136 & 62 & 8.0 & 42.5 \\
\hline D10Mit266 & 62 & D10Mit136 & 62 & 13.8 & 19.9 & D10Mit14 & 65 & 10.3 & 26.7 \\
\hline D10Mit271 & 70 & D10Mit14 & 65 & 8.4 & 35.5 & - & - & - & - \\
\hline D11Mit2 & 2 & D11Mit1 & 1 & 0.5 & 154.2 & D11Mit227 & 2 & 2.5 & 87.7 \\
\hline D11Mit271 & 21 & D11Mit236 & 20 & 14.2 & 18.4 & D11Mit242 & 31 & 4.6 & 64.1 \\
\hline D11Mit242 & 31 & D11Mit271 & 21 & 4.6 & 64.1 & D11Mit4 & 37 & 9.5 & 33.3 \\
\hline D11Mit36 & 48 & D11Mit117 & 45 & 2.1 & 101.1 & D11Mit213 & 55 & 1.3 & 105.6 \\
\hline D11Mit263 & 55 & D11Mit213 & 55 & 13.1 & 21.3 & D11Mit99 & 60 & 10.3 & 31.6 \\
\hline D11Mit224 & 66 & D11Mit166 & 64 & 6.9 & 43.1 & D11Mit214 & 74 & 1.1 & 128.0 \\
\hline D11Mit214 & 74 & D11Mit224 & 66 & 1.2 & 128.0 & D11Mit69 & 71 & 1.5 & 114.3 \\
\hline D12Mit12 & 6 & D12Mit182 & 2 & 3.0 & 76.8 & D12Mit11 & 6.0 & 8.8 & 35.8 \\
\hline D12Mit221 & 16 & D12Mit153 & 15 & 9.9 & 25.9 & D12Mit54 & 24 & 3.4 & 75.1 \\
\hline D12Mit201 & 29 & D12Mit54 & 24 & 7.4 & 45.3 & D12Mit156 & 34 & 6.1 & 54.3 \\
\hline D12Mit4 & 34 & D12Mit156 & 34 & 17.1 & 12.7 & D12Mit158 & 38 & 10.2 & 30.9 \\
\hline D12Mit259 & 45 & D12Mit204 & 41 & 5.7 & 47.8 & D12Mit97 & 47 & 6.8 & 43.9 \\
\hline D12Mit167 & 52 & D12Mit97 & 47 & 4.3 & 62.5 & D12Mit19 & 58 & 4.7 & 61.1 \\
\hline D12Mit263 & 58 & D12Mit19 & 58 & 18.0 & 10.4 & D12Nds2 & 60 & 22.1 & 27.6 \\
\hline D13Mit14 & 10 & D13Mit207 & 9 & 7.0 & 45.3 & D13Mit64 & 30 & 3.0 & 81.9 \\
\hline D13Mit64 & 30 & D13Mit14 & 10 & 3.0 & 81.9 & D13Mit139 & 32 & 6.3 & 47.6 \\
\hline D13Mit67 & 37 & D13Mit66 & 37 & 3.8 & 69.7 & D13Mit125 & 44 & 4.2 & 68.7 \\
\hline D13Mit202 & 47 & D13Mit125 & 44 & 10.3 & 31.3 & D13Mit53 & 62 & 5.2 & 59.6 \\
\hline D13Mit53 & 62 & D13Mit202 & 47 & 5.2 & 59.6 & D13Mit1 71 & 71 & 10.3 & 29.8 \\
\hline D14Mit109 & 3 & D14Mit132 & 1 & 10.0 & 26.1 & D14Mit11 & 3 & 18.6 & 6.4 \\
\hline D14Mit257 & 17 & D14Mit64 & 22 & 9.0 & 34.0 & D14Mit82 & 20 & 11.1 & 26.7 \\
\hline D14Mit158 & 29 & D14Mit82 & 20 & 2.2 & 89.7 & D14Mit203 & 29 & 14.9 & 15.9 \\
\hline D14Mit160 & 40 & D14Mit217 & 33 & 7.9 & 38.1 & D14Mit67 & 38 & 18.7 & 8.9 \\
\hline D14Mit92 & 45 & D14Mit67 & 38 & 6.2 & 55.7 & D14Mit196 & 47 & 5.4 & 49.4 \\
\hline D14Mit97 & 58 & D14Mit75 & 54 & 10.3 & 27.8 & D14Mit1 70 & 63 & 8.2 & 35.5 \\
\hline D15Mit252 & 10 & D15Mit1 79 & 11 & 1.8 & 93.1 & D15Mit136 & 14 & 3.4 & 75.7 \\
\hline D15Mit85 & 15 & D15Mit136 & 14 & 2.1 & 96.8 & D15Mit183 & 23 & 4.6 & 63.3 \\
\hline D15Mit270 & S & D15Mit183 & 23 & 7.5 & 43.9 & D15Mit29 & 43 & 5.7 & 56.2 \\
\hline D15Mit105 & 42 & D15Mit188 & 44 & 6.6 & 46.8 & D15Mit107 & 50 & 5.4 & 53.3 \\
\hline D15Mit171 & 54 & D15Mit190 & 52 & 22.9 & 3.6 & D15Mit39 & 57 & 8.7 & 36.1 \\
\hline D15Mit14 & 57 & D15Mit193 & 58 & 5.1 & 56.9 & D15Mit16 & 62 & 5.5 & 59.2 \\
\hline D16Mit146 & 17 & D16Mit145 & 14 & 10.3 & 25.6 & D16Mit57 & 22 & 5.0 & 57.6 \\
\hline D16Mit189 & 55 & D16Mit49 & 53 & 4.9 & 60.7 & D16Mit158 & 55 & 8.1 & 40.6 \\
\hline D17Mit133 & 10 & D17Mit196 & 7 & 1.9 & 97.3 & D17Mit55 & 13 & 14.3 & 14.5 \\
\hline D17Mit50 & 23 & D17Mit1 76 & 23 & 14.3 & 12.3 & D17Mit52 & 23 & 12.6 & 9.1 \\
\hline D17Mit180 & 29 & D17Mit139 & 30 & 10.3 & 29.3 & D17Mit6 & 31 & 14.9 & 16.0 \\
\hline D17Mit185 & 41 & D17Mit119 & 39 & 6.5 & 51.9 & D17Mit3 & 42 & 9.2 & 38.3 \\
\hline D17Mit72 & 47 & D17Mit39 & 45 & 8.7 & 41.1 & D17Mit73 & 49 & 11.0 & 30.6 \\
\hline D17Mit123 & 57 & D17Mit1 & 57 & 3.5 & 77.0 & - & - & - & - \\
\hline D18Mit60 & 16 & D18Mit147 & 16 & 2.7 & 81.4 & D18Mit150 & 25 & 4.1 & 74.9 \\
\hline D18Mit55 & 25 & D18Mit150 & 25 & 15.2 & 16.0 & D18Mit1 23 & 31 & 7.4 & 42.3 \\
\hline D18Mit40 & 37 & D18Mit152 & 37 & 7.2 & 41.1 & D18Mit142 & 47 & 4.5 & 58.0 \\
\hline D19Mit56 & 5 & D19Mit59 & 0 & 5.4 & 50.4 & D19Mit68 & 18 & 4.5 & 62.0 \\
\hline D19Mit111 & 15 & D19Mit79 & 6 & 5.1 & 61.6 & D19Mit40 & 25 & 6.8 & 44.3 \\
\hline D19Mit63 & 24 & D19Mit86 & 20 & 1.8 & 95.5 & D19Mit88 & 34 & 4.9 & 63.9 \\
\hline D19Mit88 & 34 & D19Mit63 & 24 & 5.0 & 63.9 & D19Mit82 & 29 & 3.4 & 73.2 \\
\hline D19Mit10 & 47 & D19Mit53 & 43 & 6.7 & 41.0 & D19Mit91 & 47 & 0.2 & 155.1 \\
\hline D19Mit6 & 55 & D19Mit71 & 54 & 15.1 & 16.6 & - & - & - & - \\
\hline DXMit54 & 2 & DXMit124 & 3 & 9.1 & 35.2 & DXMit137 & 7 & 11.0 & 25.4 \\
\hline DXMit81 & 12 & DXMit125 & 6 & 7.0 & 38.9 & DXMit49 & 14 & 9.4 & 31.1 \\
\hline DXMit192 & 19 & DXMit50 & 11 & 6.8 & 44.9 & DXMit140 & 19 & 3.5 & 62.2 \\
\hline DXMit74 & 22 & DXMit140 & 19 & 4.2 & 54.8 & DXMit1 19 & 28 & 6.6 & 36.7 \\
\hline DXMit1 70 & 29 & DXMit119 & 28 & 3.3 & 57.6 & DXMit95 & 43 & 23.0 & 1.9 \\
\hline DXMit1 72 & 40 & DXMit149 & 51 & 2.9 & 74.9 & DXMit67 & 60 & 4.9 & 57.5 \\
\hline DXMit80 & 50 & DXMit67 & 60 & 8.6 & 35.1 & DXMit179 & 62 & 6.3 & 48.9 \\
\hline
\end{tabular}

Data were analyzed using RHMAPPER (Stein et al., 1995), along with all publicly available data (http://www.jax.org). For each marker the lod scores for linkage to the next most centromeric (previous) or telomeric (next) markers are reported as well as the RH distance estimates. Also shown are the genetic map positions of the markers taken from the 1998 Chromosome Committee reports (http:// www.jax.org), except for D15Mit52 and D15Mit270, whose positions are from http://www-genome.wi.mit.edu. 
$129 / \mathrm{J}$ belongs to the M.m. domesticus group at all, as the tree is unrooted. This nominates $129 / \mathrm{J}$ as a good partner for any of the other domesticus strains in terms of polymorphism rate.

\section{RH Data}

In Table 3, RH linkage, lod score, and distance estimates are presented for each marker relative to the nearest markers in the public RH data, along with the positions of the markers on the genetic map.

The high resolution of RH mapping is a consequence of the high break frequency and also means that linkage can be clearly demonstrated only over short distances. Although lod scores are calculated for $\mathrm{RH}$ mapping in a way similar to that used for genetic mapping, the data obtained for chromosomes 17 and 13 suggests that high lod scores for linkage are also obtained between markers on different chromosomes. Based on this information, a practical lower linkage lod score limit for placement of unknown markers would be $\geq 10$. A framework having each marker linked to the next with a lod score of 10 would thus be desirable and would require a total of $\geq 1000$ mapped markers. Here we contribute 128 additional markers to this emerging framework and subdivide many of the larger intervals in the McCarthy et al. (1997) data. If independent chromosome assignment is available, the current framework can be used readily to assign markers to the intervals already defined, which correspond to $<1 \mathrm{cM}$ for chromosomes 17 and 13 and $10 \mathrm{cM}$ for the rest of the genome.

\section{METHODS}

\section{Primers}

Oligonucleotides were from TIB MOLBIOL (Berlin, Germany). The reverse primer of each pair was $5^{\prime}$ labeled with the fluorescent dye indicated in Table 1 . Many of the primers were redesigned (72 loci marked with an asterisk in the first column of Table 1; primer sequences available http:// www.mpimg-berlin-dahlem.mpg.de/ schalkwy/primer. html). The most common change was the deletion of one or more nucleotides from the $3^{\prime}$ end to avoid overlap with the microsatellite sequence and attendant stuttering artifacts.

\section{DNAs}

Mouse T31 RH panel DNAs were from Research Genetics (Huntsville, AL). C3H, SJL/J, and 129/J (stock no. 000690) DNAs were from Jackson Laboratories. PWB and C57BL/10 DNAs were a gift from J. Forejt (Czech Academy of Sciences, Prague). JF1 DNA was a gift from M. Hrabé de Angelis (GSF, Munich, Germany). C57BL/6JOlaHsd (hereafter C57BL/6), AKR/OlaHsd, and A/JOlaHsd were from Harlan Winkelmann (Borchen, Germany). BALB/cJ and DBA/2 DNAs were prepared from mice obtained from OLAC (UK).

For the mouse strain panel, PCR assays were done in a MJ tetrad cycler, using 20-sec denaturation, 20-sec annealing at the temperature given in Table 1 , and 20 -sec extension at $72^{\circ} \mathrm{C}$. Samples with different dyes were pooled and products separated on an ABI 377XL DNA sequencer using internal length standards in every lane. Analysis was with Genescan version 3.0 and Genotyper version 2.1 software from ABI.

$\mathrm{RH}$ panel assays were performed as in Schalkwyk et al. (1998): Thirty-microliter reactions with $1.5 \mathrm{~mm} \mathrm{Mg}^{2+}$ and 6.6 pmoles of each primer were amplified with a $55^{\circ}$ touchdown PCR program. For the few primers that did not give easily detectable products under these conditions, amplification was repeated with $3.0 \mathrm{~mm} \mathrm{Mg}^{2+}$. The products were detected on Southern blots of agarose gels probed with ${ }^{32} \mathrm{P}$-labeled $(\mathrm{CA})_{20}$.

For D8Mit7, RH linkage indicates that the locus is actually on chromosome 9 . Genetic mapping gives the same result (J. Walter, unpubl.). This fact may have consequences for the placement of other markers on the MIT map because D8Mit7 is identified as a framework marker.

\section{ACKNOWLEDGMENTS}

Parts of this work were funded by the Max-Planck-Gesellschaft and European Union grants BMH4-CT96-0050 (to J.W.) and CT94-0079 (to H.L.).

The publication costs of this article were defrayed in part by payment of page charges. This article must therefore be hereby marked "advertisement" in accordance with 18 USC section 1734 solely to indicate this fact.

\section{REFERENCES}

Amos, W., S.J. Sawcer, R.W. Feakes, and D.C. Rubinsztein. 1996. Microsatellites show mutational bias and heterozygote instability. Nat. Genet. 13: 390-391.

Atchley W.R. and W.M. Fitch. 1991. Gene trees and the origins of inbred strains of mice. Science 254: 554-558

Bonhomme, F. and J.-L. Guénet. 1996. The laboratory mouse and its wild relatives. In Genetic variants and strains of the laboratory mouse, 3rd ed. (ed. M.F. Lyon, S. Rastan, and S.D.M. Brown), chapter 16, pp. 1537-1576. Oxford University Press, Oxford, UK.

Breen, M., L. Deakin, B. MacDonald, S. Miller, R. Sibson, E. Tartellin, P. Avner, F. Bourgade, J.-L. Guénet, X. Montagutelli et al. 1994. Towards high resolution maps of the mouse and human genomes-A facility for ordering markers to $0.1 \mathrm{cM}$ resolution. Hum. Mol. Genet. 3: 621-627.

Deloukas, P., G.D. Schuler, G. Gyapay, E.M. Beasley, C. Soderlund, P. Rodriguez-Tomé, L. Hui, T.C. Matise, K.B. McKusick, J.S. Beckmann et al. 1998. A physical map of 30,000 human genes. Science 282: 744-746.

Dietrich, W.F., J. Miller, R. Steen, M.A. Merchant, D. Damron-Boles, Z. Husain, R. Dredge, M.J. Daly, K.A. Ingalls, T.J. O'Conner et al. 1996. A comprehensive genetic map of the mouse genome. Nature 380: 149-152.

Elliott, R.W., K.F. Manly, and C. Hohman. 1999. A radiation hybrid map of mouse chromosome 13. Genomics 57: 365-370.

Felsenstein, J. 1989. PHYLIP—Phylogeny inference package (v. 3.2). Cladistics 5: 164-166.

Festing, M.F.W. 1996. Origins and characteristics of inbred strains of mice. In Genetic variants and strains of the laboratory mouse, 3rd ed. (ed. M.F. Lyon, S. Rastan, and S.D.M. Brown), chapter 15, pp. 1537-1576. Oxford University Press, Oxford, UK.

Forejt, J., V. Vincek, J. Klein, H. Lehrach, and M. Loudová-Micková. 1991. Genetic mapping of the t-complex region on mouse chromosome 17 including the Hybrid sterility-1 gene. Mamm. Genome 1: 84-91.

Koide, T., K. Moriwaki, K. Uchida, A. Mita, T. Sagai, H. Yonekawa, H. Katoh, N. Miyashita, K. Tsuchiya, T.J. Nielsen, and T. Shiroish. 1998. New inbred strain JF1 established from Japanese fancy mouse carrying the classic piebald allele. Mamm. Genome 9: 15-19. 
Matin, A., G.B. Collin, Y. Asada, D. Varnum, D. Martone, and J. Nadeau. 1998. Simple sequence length polymorphisms that distinguish MOLF/Ei and 129/Sv inbred strains of laboratory mice. Mamm. Genome 9: 668-670.

McCarthy, L.C., J. Terrett, M.E. Davis, C.J. Knights, A.L. Smith, R. Critcher, K. Schmitt, J. Hudson, N.K. Spurr, and P.N. Goodfellow. 1997. A first-generation whole genome-radiation hybrid map spanning the mouse genome. Genome Res. 7: 1153-1161.

Primmer, C.R., N. Saino, A.P. Møller, and H. Ellegren. 1998.

Unraveling the processes of microsatellite evolution through analysis of germ line mutations in barn swallows Hirundo rustica. Mol. Biol. Evol. 15: 1047-1054.

Schalkwyk, L.C., M. Weiher, M. Kirby, B. Cusack, H. Himmelbauer, and H. Lehrach. 1998. Refined radiation hybrid map of mouse chromosome 17. Mamm. Genome 9: 807-811.

Silver, L.M. 1995. Mouse genetics: Concepts and applications. Oxford University Press, Oxford, UK.

Stein, L., L. Kruglyak, D. Slonim, and E. Lander. 1995. "RHMAPPER," unpublished software, Whitehead Institute/MIT Center for Genome Research. Available at http://www.genome.wi.mit.edu/ $\mathrm{ftp} / \mathrm{pub} /$ software/rhmapper/ and ftp.genome.wi.mit.edu, directory/pub/software/rhmapper.

Weber, J. and C. Wong. 1993. Mutation of human short tandem repeats. Hum. Mol. Genet. 2: 1123-1128.

Received May 12, 1999; accepted in revised form July 8, 1999. 


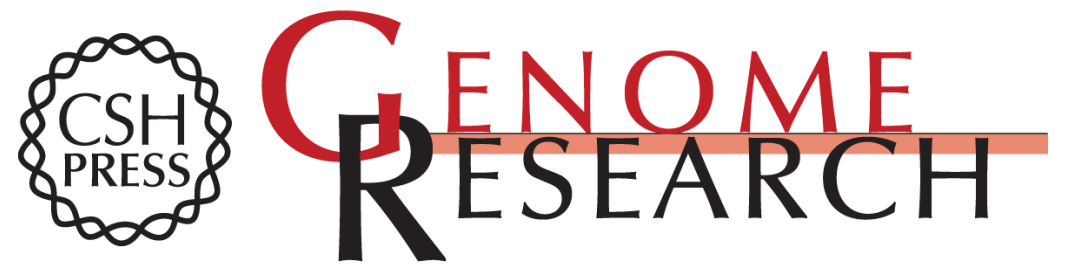

\section{Panel of Microsatellite Markers for Whole-Genome Scans and Radiation Hybrid Mapping and a Mouse Family Tree}

Leonard C. Schalkwyk, Martin Jung, Angelika Daser, et al.

Genome Res. 1999 9: 878-887

Access the most recent version at doi:10.1101/gr.9.9.878

References This article cites 14 articles, 3 of which can be accessed free at:

http://genome.cshlp.org/content/9/9/878.full.html\#ref-list-1

\section{License}

Email Alerting Receive free email alerts when new articles cite this article - sign up in the box at the Service top right corner of the article or click here.

\section{Affordable, Accurate Sequencing.}

